\title{
The effect of angle of attack on the aeroacoustic environment within the weapons bay of a generic UCAV
}

\author{
David Bacci, Alistair J. Saddington ${ }^{1}$, Derek Bray \\ Aeromechanical Systems Group, Centre for Defence Engineering, \\ Cranfield University, Defence Academy of the UK, Shrivenham, SN6 8LA, UK
}

\begin{abstract}
Cavity flow studies are generally concerned with observing the effect of geometry changes whilst maintaining a fixed zero angle of attack. Cavities employed as weapons bays will, however, experience a range of angles of attack. This paper presents the first known results showing the effect of flight angle of attack on the aeroacoustic characteristics of an internal weapons bay installed in an uninhabited combat air vehicle (UCAV). The UCAV geometry consisted of a Boeing M219-type cavity in a Boeing UCAV1303 airframe. Numerical simulation was conducted using a full-scale detached eddy simulation model and representative transonic flight conditions. As well as the reference case of zero degrees, data for angles of attack of 3.0, 4.5 and 6.0 degrees were analysed. Experimental data was used to validate the reference computational model, which agreed with the overall fluctuating sound pressure level (OAFPL) to within the experimental uncertainty of $4 \mathrm{~dB}$. Data from the computational model was post-processed with frequency-domain and time-frequency-domain techniques showing that the flow structure within the weapons bay was altered significantly by the angle of attack changes, affecting the mean pressure distribution, frequency spectra and resonant modes. Overall, increasing the angle of attack from 0.0 to 3.0 degrees produced an increment in the acoustic load whilst a further increase tended to affect the resonance mechanism and thereby reduce the coherence and the temporal footprints of the resonant modes.
\end{abstract}

\footnotetext{
Email address: a.j.saddington@cranfield.ac.uk (Alistair J. Saddington)

${ }^{1}$ Corresponding author.
}

Preprint submitted to Aerospace Science and Technology

fuly 22, 2019 
Keywords: UCAV; cavity; aeroacoustic; transonic

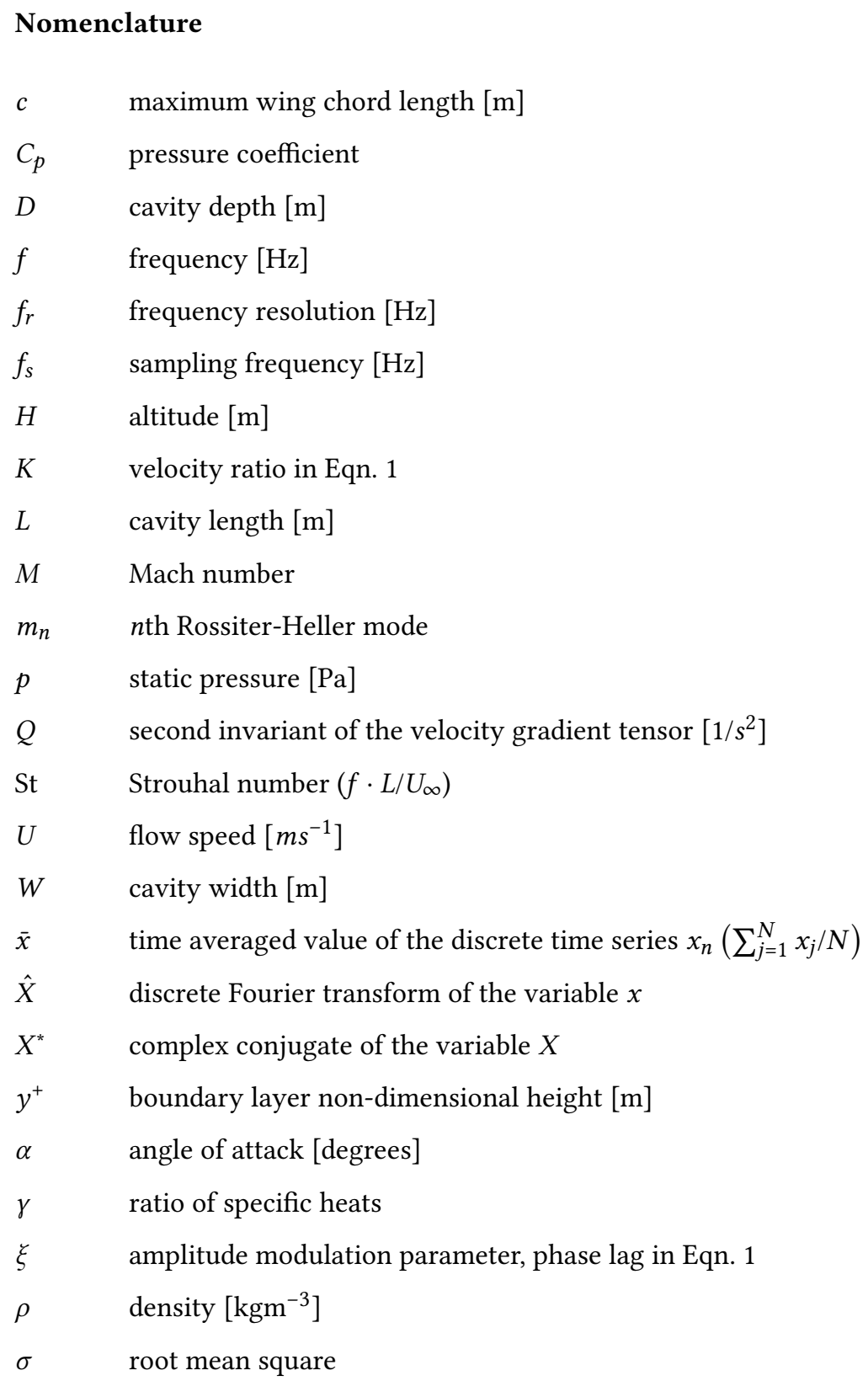




$\begin{array}{ll}\text { Abbreviations } \\ \text { AOA } & \text { angle of attack } \\ \text { CFD } & \text { computational fluid dynamics } \\ \text { CFL } & \text { Courant-Friedrichs-Lewy condition } \\ \text { DES } & \text { detached-eddy simulation } \\ \text { DFT } & \text { discrete Fourier transform } \\ \text { IDDES } & \text { improved delayed detached-eddy simulation } \\ \text { ISA } & \text { International Standard Atmosphere } \\ \text { LIC } & \text { line integral convolution } \\ \text { OASPL } & \text { overall sound pressure level [dB] } \\ \text { PR } & \text { persistence index (of a signal) } \\ \text { PSD } & \text { power spectral density } \\ \text { RCS } & \text { radar cross section } \\ \text { SPL } & \text { sound pressure level [dB] } \\ \text { UCAV } & \text { unmanned combat air vehicle } \\ \text { WSP } & \text { wavelet spectral power [dB] } \\ & \\ \text { Subscript } & \\ \text { e } & \text { edge of boundary layer } \\ \infty & \text { freetsream conditions }\end{array}$

\section{Introduction}

Modern fifth generation combat aircraft are often required to have a reduced radar cross-section (RCS) when compared to earlier generations and this has introduced the need to incorporate internal weapons bays into their design. When the bay doors are opened the flow in the bay becomes highly turbulent and unsteady which has the potential to jeopardise the safe release of weapons. Moreover, airframe and weapons can be damaged by the intense acoustic field generated by the flow with unsteady pressure levels of up to $170 \mathrm{~dB}$ at certain resonant modes. The acoustic phenomena are strongly dependant on the geometry and freestream conditions [1] and may be 
observed under subsonic [2] and supersonic flow conditions [3-5]. The frequencies at which resonance occurs are called Rossiter modes, following his early work on cavity flows [6]. The mode frequencies are more generally predicted using the Rossiter-Heller equation (Eqn. 1), which accounts for the higher speed of sound within the cavity at high freestream Mach numbers Heller et al. [7]. Here,

$$
\mathrm{St}_{n}=\frac{n-\xi}{M_{\infty}\left[1+\frac{(\gamma-1)}{2} M_{\infty}^{2}\right]^{-\frac{1}{2}}+\frac{1}{K}}
$$

where $S t_{n}$ is the Strouhal number corresponding to the $n$th mode, $K$ is the ratio of disturbance velocity in the shear layer to the freestream velocity (generally taken as $0.57)$ and $\xi$ is an empirical constant employed to account for the phase lag between the passage of a vortical disturbance past the cavity trailing edge and the formation of an upstream travelling disturbance ${ }^{2}$. The value of $\xi$ depends on the length-to-depth ratio, $L / D$, of the cavity and is evaluated as $\xi=0.062(L / D)$ [6]. The ratio of specific heats, $\gamma$, is assumed to be equal to 1.4 for air.

A subsequent study by Delprat [8] introduced a new model for the resonating mechanism, which explained the additional peaks visible in the frequency spectra as well as the main Rossiter modes. It was observed that the Rossiter-Heller tones could be interpreted as the result of a frequency shift (i.e. an amplitude modulation) of a frequency-modulated wave. Delprat [8] proposed the following equation for a generic Rossiter tone of $n$th order $\left(f_{n}\right)$.

$$
f_{n}=(n-\xi) \cdot\left[f_{a} \pm k \Delta f\right] \quad n=1,2,3, \ldots
$$

In Eqn. $2, f_{a}$ is the fundamental frequency loop of the cavity (also called the "carrier frequency'); $\xi$ is the amplitude modulation parameter, or the ratio of the amplitude modulation frequency, $f_{b}$, to $f_{a}$; $\Delta f$ is the modulating frequency (which usually coincides with the lowest frequency peak in the spectrum); and $k$ is an integer. From this supposition, Delprat [8] derived the pseudo-harmonic approximation. Hence, the

\footnotetext{
${ }^{2}$ In their original papers Rossiter [6] used $\gamma$ for the phase lag parameter, whilst Heller et al. [7] used $\alpha$. In this paper we use $\xi$ to avoid confusion with the ratio of specific heats $(\gamma)$ and the angle of attack $(\alpha)$.
} 
Rossiter tones can be deduced from a knowledge of the carrier frequency, $f_{a}$, the modulating frequency, $\Delta f$, and the amplitude-modulation frequency, $f_{b}$.

Knowing that in its formulation the Rossiter-Heller equation does not take into account the modulating frequency $\Delta f$, it is possible to deduce that the amplitude modulation parameter, $\xi$, is a function of $L / D$,

$$
\xi=\frac{f_{b}}{f_{a}}=0.062\left(\frac{L}{D}\right)
$$

and that the fundamental frequency loop of the cavity is correlated to the freestream Mach number, $M_{\infty}$, and the feedback loop constant, $1 / K$, as

$$
\mathrm{St}_{a}=\frac{1}{M_{\infty}\left[1+\left(\frac{\gamma-1}{2}\right) M_{\infty}{ }^{2}\right]^{-\frac{1}{2}}+\frac{1}{K}}
$$

Despite this knowledge, it is difficult to extrapolate simple experimental results to the design of weapons bays installed in real-life combat aircraft because the bays are generally not rectangular and the surrounding environment is complex. Some work has been published in the open literature concerning the analysis of existing manned combat aircraft designs [9-11] and unmanned combat air vehicle (UCAV) platforms with a weapons bay installed [12-14]. Further studies have addressed some of the geometric features of current designs, such as bay doors, indented leading and trailing edges, and contoured surfaces in front of the cavity [15-17]. One parameter that has not been discussed in the literature is the effect of angle of attack on cavity flows. In combat, military aircraft may release a store at non-zero angles of attack. Nevertheless, the standard approach to the analysis of cavity flows, even when installed in airframes, has been to fix the angle of attack seen by the cavity to zero in order to explore the effects of other parameters.

This article focuses on the effect of varying angle of attack on the behaviour of a representative weapons bay and airframe combination exposed to a transonic freestream flow. The geometry consisted of a Boeing M-219-type cavity installed in a Boeing UCAV1303 airframe. The numerical analysis uses a full-scale aircraft model, rather than a scaled wind-tunnel geometry. Numerical simulation was achieved using the detached eddy simulation (DES) turbulence model, which has been demonstrated to 
be appropriate for use with cavity flows $[12,13]$. The angles of attack analysed, in addition to the reference configuration at 0.0 degrees, were 3.0, 4.5, and 6.0 degrees. All simulations were executed at a flight Mach number of 0.85 with the bay doors open at 90 degrees.

\section{Numerical method}

\subsection{Geometry}

The geometry was based on the Boeing/AFRL 1303. This is a generic UCAV with a blended wing body (BWB) layout, two Boeing/M219-type weapons bays. The Wshaped planform has a leading edge sweep of 47 degrees, an overall length of $10 \mathrm{~m}$, and a wingspan of $16.5 \mathrm{~m}$. To simplify the UCAV model for computational fluid dynamics (CFD) and wind tunnel testing, the air intakes and engine exhausts are not included in the geometry. The M219 weapons bay is a rectangular cavity with $L / D=5$ and a width-to-depth ratio $(W / D)$ of one. It has been used extensively as a standard model for the analysis of transonic cavity flows. However, its installation on the airframe of the UCAV 1303 requires some geometry modifications. The underside of the aircraft is not flat but has a mean slope of 3.6 degrees from the centreline towards each wing tip. Moreover, to accommodate safely a GBU-39 store the cavity was stretched in its transverse and longitudinal dimensions, resulting in a variant of the standard M219 cavity geometry, here designated M219-Mod000. The resulting CFD model (Fig. 1) was simplified further by removing the port side weapons bay.

The reference axis system was right-handed, with its origin at the UCAV nose on the symmetry plane. The positive $x$-axis points downstream, the positive $y$-axis along the starboard wing and the positive $z$-axis upwards (Fig. 1). The far field was an ellipsoid centred at the origin with semi-axes in the $x-y-z$ directions of $25 c, 10 c$ and $10 c$, respectively. These dimensions were taken from earlier validated studies [12] and therefore no further domain sensitivity study was conducted. The upstream and downstream edges of the cavity are not parallel to the cavity floor but inherit the slope of the airframe underside, whereas the cavity floor remains unchanged and flat. The bay was accompanied by two doors, with double 45 degree saw-toothed leading and 


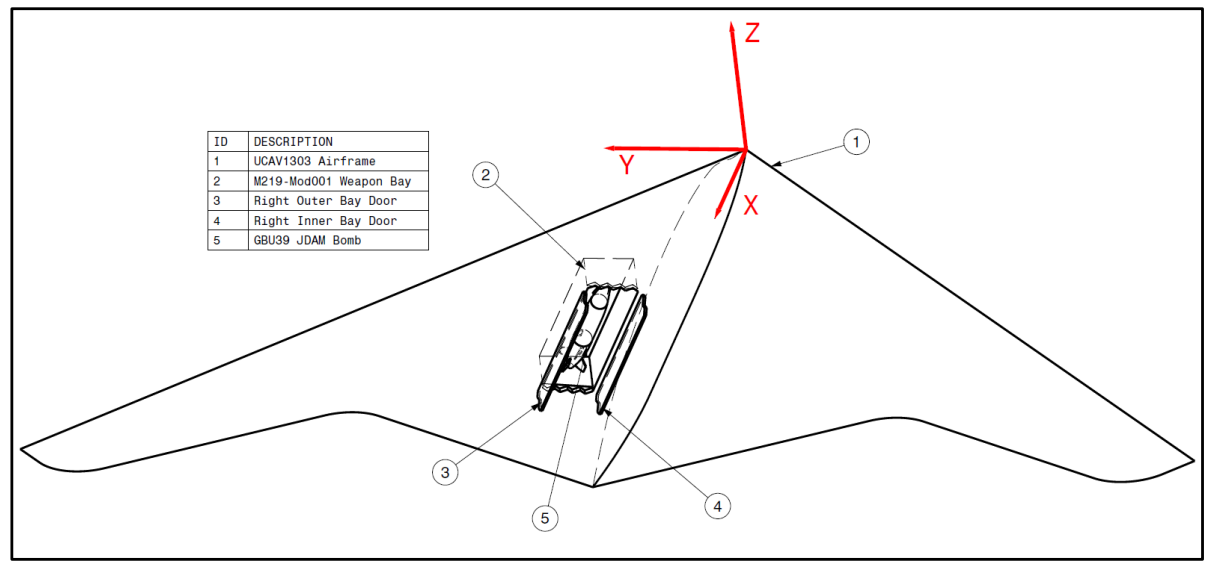

Figure 1: Solid model of the UCAV 1303 and M219-Mod000 configuration.

trailing edge profiles. For the current study, the doors were fixed open at 90 degrees. The detailed geometry and principal dimensions are shown in Fig. 2.
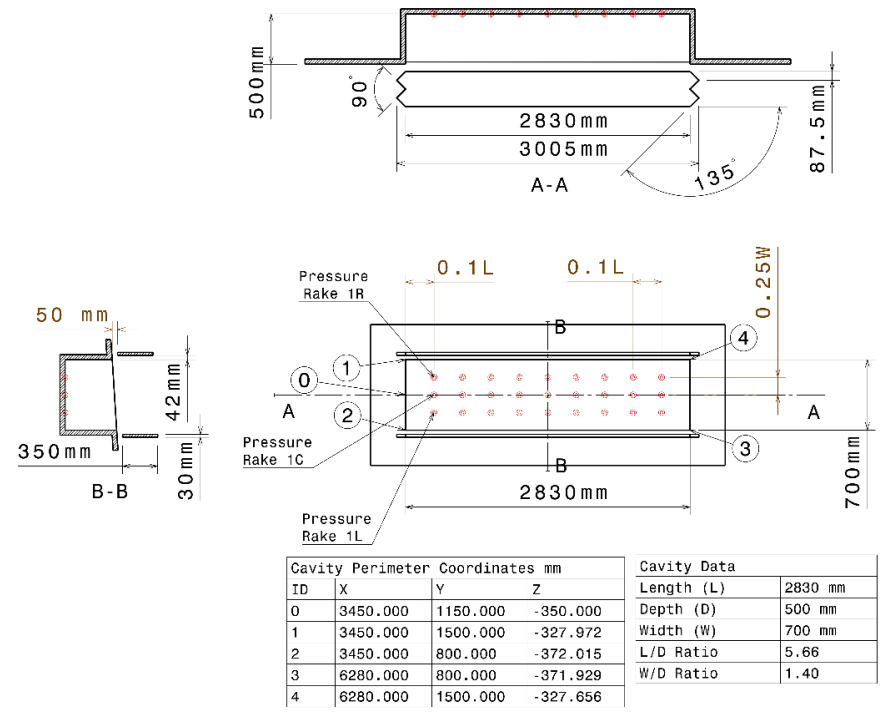

Figure 2: Details of the M219-Mod000 cavity showing the principal dimensions and the location of the pressure sample points along rakes, $1 \mathrm{R}, 1 \mathrm{C}, 1 \mathrm{~L}$. 


\begin{tabular}{|c|c|c|c|c|c|c|}
\hline & $x(\mathrm{~m})$ & $y(\mathrm{~m})$ & $z(\mathrm{~m})$ & $x / L$ & $2 y / W$ & $z / D$ \\
\hline \multirow[t]{2}{*}{ Bay origin } & 3.450 & 1.150 & -0.350 & 0.0 & 0.0 & 0.0 \\
\hline & 3.733 & & & 0.1 & & \\
\hline \multirow[t]{3}{*}{ Rake 1L } & 4.016 & 0.800 & & 0.2 & -0.5 & \\
\hline & 4.299 & & & 0.3 & & \\
\hline & 4.582 & & & 0.4 & & \\
\hline \multirow[t]{3}{*}{ Rake $1 \mathrm{C}$} & 4.865 & 1.150 & 0.150 & 0.5 & 0.0 & 1.0 \\
\hline & 5.148 & & & 0.6 & & \\
\hline & 5.431 & & & 0.7 & & \\
\hline \multirow[t]{2}{*}{ Rake 1R } & 5.714 & 1.500 & & 0.8 & 0.5 & \\
\hline & 5.997 & & & 0.9 & & \\
\hline
\end{tabular}

Table 1: Coordinates of the rakes used to record pressure data.

A total of 27 probes were placed inside the cavity to monitor the static pressure used for the frequency analysis (Fig. 2). They were assigned to three longitudinal rakes with a non-dimensionalised local coordinate system shown in Table 1. Note that $x / L=0$ indicates the cavity front face, $2 y / W=0.0$ indicates the cavity centreline, and $z / D=0$ indicates the cavity exit plane. Data was sampled at $10 \mathrm{kHz}$. Time histories were time-moving, averaged with a rectangular window of 10 samples and $50 \%$ overlap to remove any aliasing effect that could have been introduced in the computation.

\subsection{Numerical simulation}

DES is a turbulence model that automatically blends Reynolds-averaged NavierStokes (RANS) and large eddy simulation (LES) modelling. The DES model has no practical limit to the fineness of the computational grid because the finer the mesh, the smaller the size of the eddies that are resolved instead of modelled [18]. The boundary layer state at the leading edge of the bay influences its aeroacoustic behaviour [19]. The geometry upstream of the bay edge must be meshed adequately, therefore, to ensure accurate resolution by the RANS model. Given the complex nature of the problem, an 
unstructured tetrahedral-type mesh was used with a prism layer wrapped around the viscous surfaces for accurate boundary layer resolution. The critical areas were the shear layer, detaching from the cavity leading edge, and the flow inside the weapons bay. For accurate shear layer modelling with DES, Menter [20] suggests a mesh spacing of at least $1 / 5$ of the local boundary layer thickness, with 1/20 being an ideal value. In this study, a value of $1 / 15$ was chosen which gave a good compromise between computational cost and the resulting data quality. The prism layers, enveloping all viscous surfaces, comprised 29 layers with an expansion ratio of 1.3 (as suggested by Spalart [18]), and an initial $y^{+}$value of 0.5 , as suggested by Wilcox [21]. The simulations were performed under environmental conditions of Mach 0.85 and an altitude of $9144 \mathrm{~m}$ $(30,000 \mathrm{ft})$ in an international standard atmosphere (ISA). All solid walls were specified as adiabatic surfaces where the no-slip velocity condition $(u=v=w=0)$ was applied. The computational outer boundary was specified as a far-field using the modified Riemann invariants condition.

The simulations in this study were performed using the IDDES model within the ANSYS Fluent R16.2 CFD code [22]. This implementation uses the $k-\omega$ SST turbulence model [23] with the IDDES modifications described by Shur et al. [24]. The air was treated as an ideal compressible gas using Sutherland's law for viscosity modelling. Non-viscous fluxes were resolved using a third-order MUSCL scheme; viscous fluxes were resolved using a second-order upwind scheme; and gradients were evaluated using the least squares method. Due to the compressible nature of the problem the density-based solver was used. The time marching scheme was an implicit secondorder dual-time formulation. Five inner sub-iterations were found to be sufficient, which is consistent with the recommendation of Menter [20] and the approach of Chaplin and Birch [13]. As advised by Menter [20], the time step $\left(1.0 \times 10^{-5} \mathrm{~s}\right)$ was chosen to ensure a CFL number approximately equal to unity in the LES part of the domain. Unsteady simulations were initiated from a partially-converged RANS model to reduce the start-up time. To resolve accurately all the main features, a simulation duration of at least 30 cycles of the frequencies of interest is required [20]. In the present case, the aim was to resolve the physical problem up to the lowest RossiterHeller mode, estimated from Eqn. 1 to be $23 \mathrm{~Hz}$. This gave a minimum simulation time 


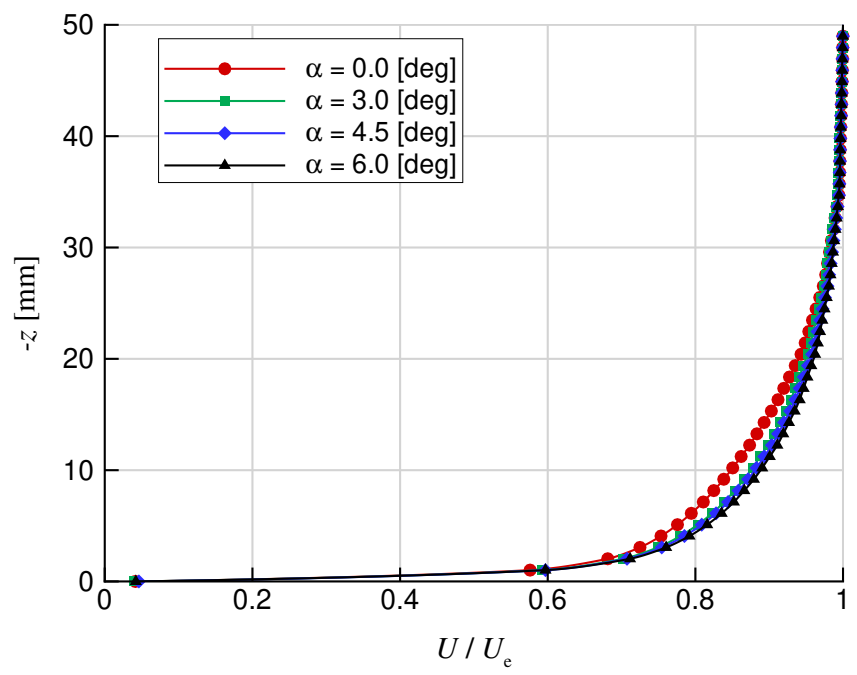

Figure 3: Boundary layer profiles at the leading edge centreline of the weapons bay.

of $1.3 \mathrm{~s}$. In practice simulations were run for $220 \times 10^{3}$ iterations $(2.2 \mathrm{~s})$, discarding the first $0.2 \mathrm{~s}$ to clear the solution from the start-up transient. This provided a total of 46 cycles of the lowest frequency mode.

The onset boundary layer at the leading edge centreline of the weapons bay is shown in Fig. 3 with all four profiles approximating closely to a seventh power law. The observed differences are due to the variation in the presented profile of the UCAV's lower surface to the freestream flow with respect to both the surface length over which the boundary layer grows and the degree of acceleration. Increasing angle of attack gives a shorter growth length and less acceleration resulting in the boundary layer thickness only varying by $\pm 1 \mathrm{~mm}$ about a mean of $32.6 \mathrm{~mm}$. Based on previous observations by Thangamani et al. [19], where much larger changes in boundary layer thickness were required to produced measurable changes in cavity flow behaviour, the change in thickness noted here was not deemed significant enough to correlate with any changes in the flow structure within the weapons bay.

To validate the computational settings and mesh convergence, data were used from wind tunnel studies on a 1:10.8 scale model published by Chaplin and Birch [13]. The CFD model was scaled to the wind tunnel geometry and the test conditions replicated. 


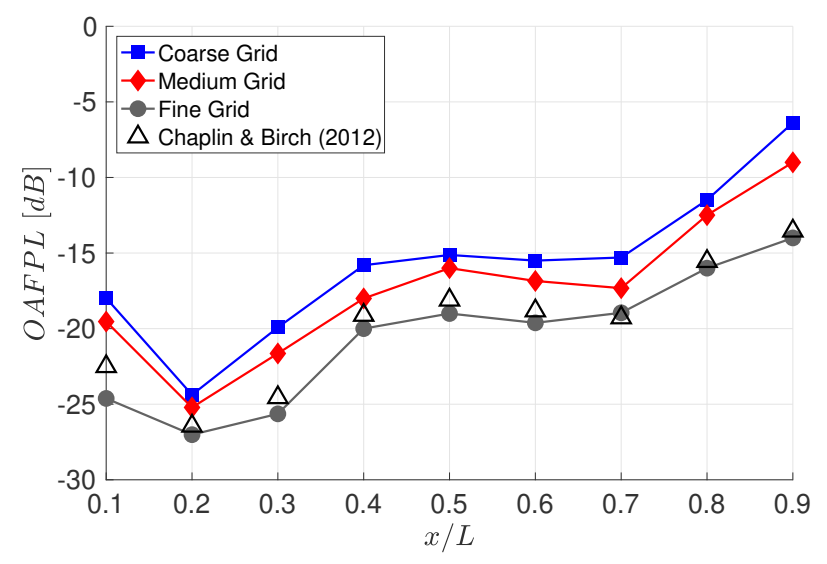

Figure 4: CFD grid convergence on OAFPL.

Grid convergence was stopped when the numerical results showed a difference in the overall fluctuating pressure level (OAFPL) ${ }^{3}$, with respect to the wind-tunnel measurements, at each of the nine measurement locations of less than $4 \mathrm{~dB}$ (Fig. 4). This placed the numerical results within the error band of the experimental data [12]. For the fine grid used in this study the difference was in practice less than $2 \mathrm{~dB}$. This level of precision was considered satisfactory for the current study, which sought to investigate trends relating to the effects of angle-of-attack changes. The final step was to adapt the wind-tunnel mesh settings to the full-size models. This resulted in the construction of a mesh containing 24 million elements, of which ten million were inside the weapons bay.

\section{Data analysis procedures}

The triple decomposition approach of Hussain [26] was used to analyse the pressure histories. The signal is divided into a time-mean component (mean flow analysis), and an unsteady component (non-stationary flow analysis). The latter is further di-

\footnotetext{
${ }^{3}$ The OAFPL is a measure of the ratio of the root mean square of a pressure signal and the freestream dynamic pressure. This quantity was first introduced by Tracy and Plentovich [25] to compare the OASPL of cavities tested under different environmental conditions.
} 
vided into a coherent component (resonant modes) and an incoherent component (broadband noise due to turbulence). The mean flow analysis consisted of the study of the time-averaged pressure coefficient, $\overline{C_{p}}$, and the overall sound pressure level (OASPL). These quantities were defined, respectively, as,

$$
\overline{C_{p}}=\left[\frac{2}{\gamma M_{\infty}^{2}}\left(\frac{\bar{p}}{p_{\infty}}-1\right)\right]
$$

and,

$$
\text { OASPL }=20 \log _{10}\left(\frac{\sigma_{p}}{p_{\text {ref }}}\right)
$$

Here $p_{\text {ref }}$ is the minimum audible pressure, equal to $2 \times 10^{-5} \mathrm{~Pa}$, which is defined as the threshold of human hearing.

The non-stationary flow analysis involved both frequency and time-frequency domains. For the frequency domain analysis the sound pressure level (SPL) of the fluctuating part of the pressure signal was calculated as [27],

$$
\operatorname{SPL}(f)=10 \log _{10}\left(\frac{\operatorname{PSD}_{p_{f}}(f) \cdot \Delta f_{\text {ref }}}{p_{\text {ref }}^{2}}\right)
$$

where, $\Delta f_{\text {ref }}$ is a reference frequency set equal to $1 \mathrm{~Hz}$. In this study the power spectral density (PSD) was obtained using the Welch method [28]. The original signal was divided into smaller blocks with $50 \%$ overlap. The number of samples was equal to the ratio $f_{s} / f_{r}$, where $f_{s}$ is the sampling frequency $(10 \mathrm{kHz})$ and $f_{r}$ is the frequency resolution $(2 \mathrm{~Hz})$. A Hanning window was then applied to each block to reduce leakage effects. Finally, the PSD of the original signal was calculated by averaging the PSD of each block.

For the time-frequency domain analysis, the Morlet wavelet transform was used to calculate the wavelet spectral power (WSP) map [27] where,

$$
\mathrm{WSP}(s, n)=10 \log _{10} \frac{W(s, n) \cdot W^{*}(s, n)}{p_{\mathrm{ref}^{2}}} \cdot \frac{\mathrm{d} t}{s}
$$

Here, $W(s, n)$ is the wavelet transform of the fluctuating part of the pressure signal; $\mathrm{d} t$ is the time step at which the signal was recorded; and $s$ represents the wavelet scales.

For the characterisation of the statistical non-stationary modes the index of persistence (PR) was used [27]. This index varies from $0 \%$, indicating that a particular 
tone is inactive during the time window, to $100 \%$, indicating that a particular tone is always active during the time window.

The uncertainty of the measurements was calculated using the methodology derived by Bruun [29], which gave a maximum error on the mean quantities of $1.2 \%$ with a confidence level of $95 \%$. The uncertainty in PSD was estimated using the procedure described by Newland [30], resulting in a precision of $-1.9 \mathrm{~dB}$ to $+2.6 \mathrm{~dB}$ with a confidence level of $95 \%$. Finally, for the estimation of the wavelet transform's uncertainty, the method described by Torrence and Compo [31] was used.

\section{Results}

The results were divided into time-mean and non-stationary (i.e. unsteady) flow analyses. Mean pressure, mean velocity, and OASPL values were analysed to characterise the mean flow properties. The non-stationary components were obtained by extracting information with the Fourier and wavelet transforms.

\subsection{Mean flow}

The longitudinal mean pressure coefficient, at zero angle of attack (Fig. 5), showed the typical trend of a transitional-open-type cavity, with a small negative gradient up to $x / L=0.4$ in which $\overline{C_{p}}$ was always negative (ranging from -0.22 to -0.25 ). This was followed by a rise towards $\overline{C_{p}}=0$, in which the curve changes from a concave to a convex shape. The introduction of a positive angle of attack added a vertical velocity component to the freestream velocity, directed towards the cavity interior. The effect of changing the angle of attack from 0.0 to 3.0 degrees, and from 3.0 to 6.0 degrees, was to cause a vertical shift of the curve towards more positive $\overline{C_{p}}$ values, equating to approximately 0.1 units every 3 degrees. An unexpected trend was, however, encountered at 4.5 degrees angle of attack. Here, only stations up to $x / L=0.5$ experienced the general increase in $\overline{C_{p}}$; in the rear half of the bay the pressure coefficient was approximately equal to that obtained for the 3.0 degrees angle-of-attack case. This suggests that changes in angle of attack have a non-monotonic influence in the cavity flow behaviour. 


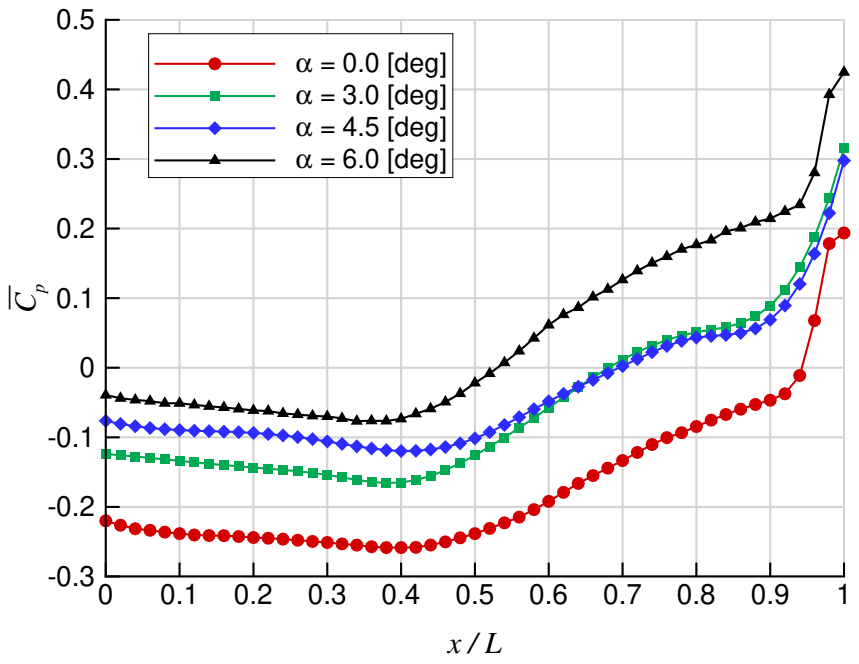

Figure 5: The streamwise variation of mean pressure coefficient at four angles of attack $(2 y / W=0)$.

The distribution of OASPL along the length of the cavity is shown in Fig. 6. While the general shape was always similar to the reference case ( $\alpha=0$ degrees), exhibiting a concave-convex-concave form with inflection points located at $x / L \approx 0.2$ and $x / L \approx 0.6$, the effect of changing angle of attack was to shift the curves to different levels of OASPL. An angle of attack change from 0.0 to 3.0 degrees increased OASPL by around $1 \mathrm{~dB}$ at all stations. This result correlates to an increase in the freestream flow being directed into the cavity, thus adding more energy to the pressure oscillations. However, increasing the angle of attack from 3.0 to 4.5 degrees reversed this trend. Here, the signal power was lower than the reference case by an average of $2 \mathrm{~dB}$ across the whole cavity, suggesting a change to the flow characteristics and noise accumulation mechanism. Increasing the angle of attack from 4.5 to 6.0 degrees restored the original trend and the OASPL was raised to a level similar to that obtained with the reference case. These results suggest that the cavity flow undergoes a strong reconfiguration as the angle of attack is increased.

To investigate the observed behaviour in $\overline{C_{p}}$ and OASPL further, the mean velocity vector field was visualised using line integral convolution (LIC) [32]. This revealed a complex series of flow structures, that changed with angle of attack. Fig. 7a shows LIC 


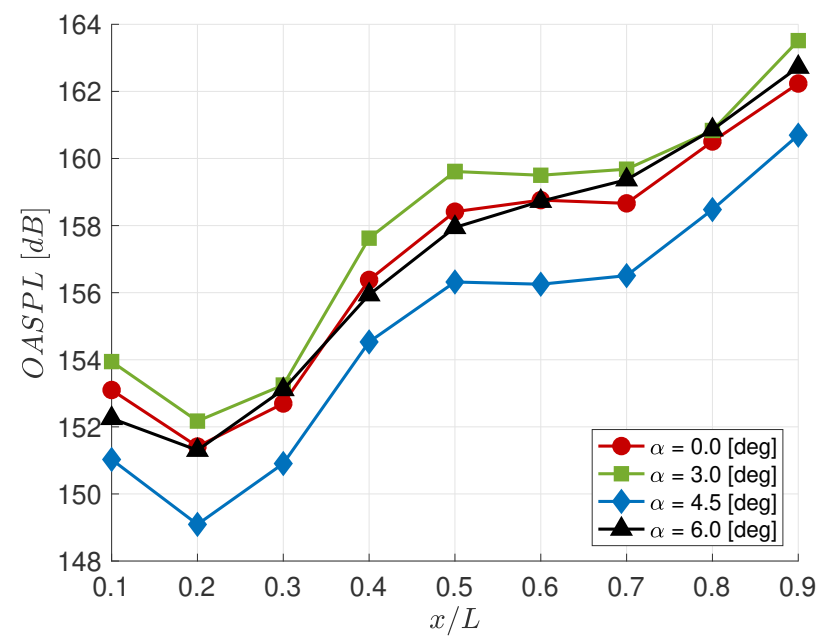

Figure 6: The streamwise variation of OASPL at four angles of attack $(2 y / W=0)$.

images for the reference configuration ( $\alpha=0$ degrees) in the $x-y$ plane with the main features identified. The flow field is characterised by a large clockwise recirculation zone, centred at approximately $x / L=0.42$, and two additional vortices close to the cavity floor. The front vortex has a counter-clockwise sense of rotation, while the rear one is clockwise. The shear layer, that develops following separation of the freestream flow from the front wall of the cavity, impinges on the rear wall at $z / D \approx 0.1$. The fluid above the rear stagnation point is ejected from the cavity over the rear wall and joins the freestream flow. The fluid below the shear layer impingement point is deflected downwards towards the bottom of the cavity. Some of this flow is entrained into the rear vortex, however, the majority forms the main recirculation zone, which is observed to be sufficiently large as to extend its influence to the front wall of the cavity where it interacts with the shear layer formation. The centre of the main recirculation zone does not coincide with the location of the minimum value of $\overline{C_{p}}$ shown in Fig. 5; the pressure minima is approximately $0.04 x / L$ forward of the core of the recirculation zone. For a circular vortex close to a surface we would expect the location of the vortex core to correlate with the pressure minima. However, in the present study the recirculation zone is distorted by varying amounts in both axes in the $\mathrm{x}-\mathrm{z}$ plane. As a consequence, 
the longitudinal positions of the vortex core and pressure minima are not aligned.

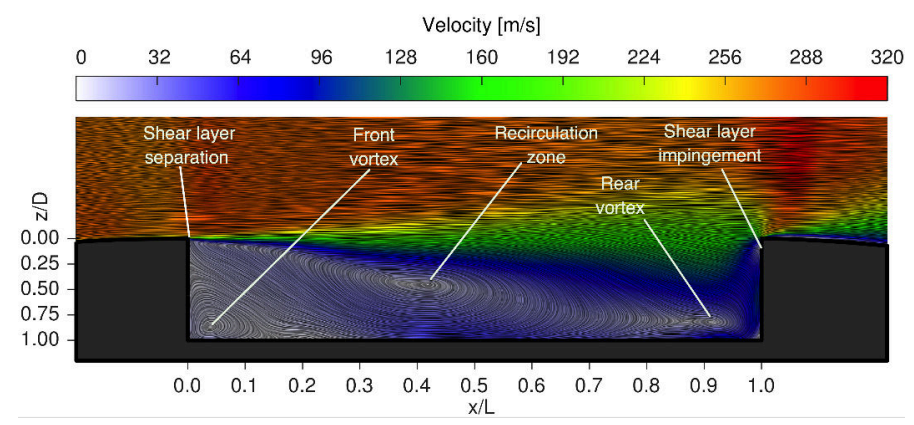

(a) $x-z$ plane, $2 y / W=0.0$

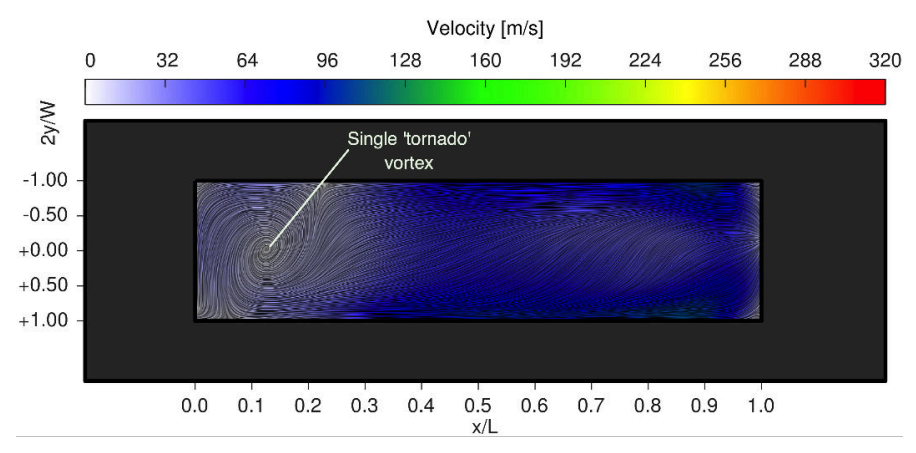

(b) $\quad x-y$ plane, $z / D=0.5$

Figure 7: The mean velocity field at $\alpha=0.0$ degrees; flow from left to right.

Fig. 7b shows that the flow field in the $x-y$ plane is characterised by a large clockwise 'tornado'-like vortex, centred at $x / L \approx 0.13$, and fed by the upstream flow at this height. Its streamwise location appears to coincide approximately with the region where the reversed flow on the floor of the cavity is deflected upwards towards the shear layer by the front vortex. Overall, the flow in the $x-y$ plane is asymmetrical about the centreline plane $(2 y / W=0.0)$. This is due, in part, to the low value of $W / D$, which has been observed to show asymmetry in the mean flow [33], and also due to the asymmetric cavity geometry and spanwise pressure gradient in the $y-z$ plane. At larger values of $W / D$ a symmetrical pair of contra-rotating tornado vortices are usually expected [34, 35]. As observed by Khanal et al. [34], the axis of the tornado vortex is turned from a $z$-direction orientation to an $x$-direction orientation before 


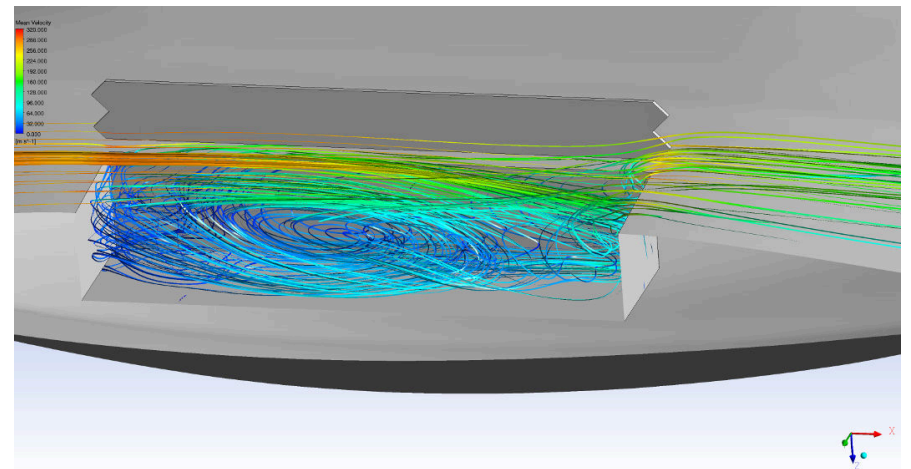

Figure 8: Three dimensional visualisation of the cavity flow using stream ribbons coloured by velocity magnitude ( $\alpha=0$ degrees); to aid visualisation the aircraft structure, including the door, outboard of the $x-z$ plane at $2 y / W=1$ has been removed.

exiting the cavity and merging with the freestream flow. A typical example is shown in Fig. 8.

Fig. 9a shows LIC images for the flow field at $\alpha=3.0$ degrees. Compared with the reference case the flow-field between the front wall and the main vortex is changed by the addition of a small counter-clockwise secondary vortex, located at $x / L \approx 0.25$, beneath the main recirculation zone. The main recirculation zone has reduced in size, particularly with respect to its forward extent, sits slightly lower, and has its core slightly further forward $(x / L=0.40)$ than was the case at $\alpha=0.0$ degrees. It is evident from the velocity contours that with a positive angle of attack higher velocity fluid is being entrained into the cavity, however, the rear stagnation point remains at $z / D \approx 0.1$. The minimum value of $\overline{C_{p}}$ is now approximately $0.02 x / L$ ahead of the core of the recirculation zone.

In the $x-y$ plane (Fig. 9b) the flow configuration is completely altered with a multiple eddy system now present. There are two contra-rotating 'tornado'-like vortices at $x / L \approx 0.16$ (clockwise) and $x / L \approx 0.25$ (counter-clockwise); the forward one is the stronger and more dominant of the two. These appear to emanate, respectively, from the upwards motion of fluid between the front and secondary vortices, and from the flow in front of the main recirculation zone. Behind these vortices flow is moving upstream at this height. 


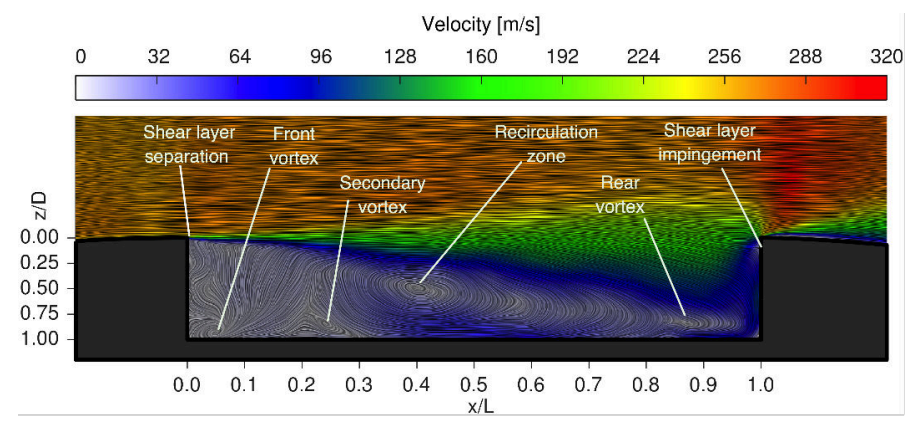

(a) $x-z$ plane, $2 y / W=0.0$

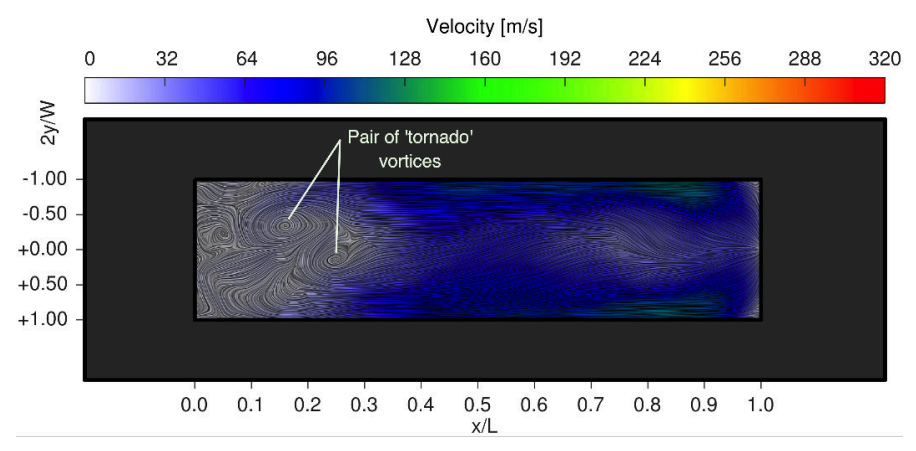

(b) $x-y$ plane, $z / D=0.5$

Figure 9: The mean velocity field at $\alpha=3.0$ degrees; flow from left to right.

With a further increase in angle of attack (from 3.0 to 4.5 degrees), the flow topology again changes. Fig. 10a shows that in the $x$ - $z$ plane the main clockwise vortex is again reduced in size and is located more towards the front of the cavity $(x / L=0.38)$. This is due to the increased inward deflection of the shear layer, which now creates a stagnation point on the cavity floor at $x / L \approx 0.83$. The main vortex changes to an elliptical form, due to the combined action of stretching by the shear layer, and constraint by the cavity floor. The consequence of this is that the minimum value of $\overline{C_{p}}$ is now approximately $0.03 x / \mathrm{L}$ aft of the core of the recirculation zone. For cases in which the angle of attack was 0.0 and 3.0 degrees, the vortex's major axis is almost horizontal. However, for $\alpha=4.5$ degrees, the major axis is tilted downwards towards the rear by approximately 30 degrees.

In the $x-y$ plane (Fig. 10b) a single 'tornado-like' vortex structure reappears in 


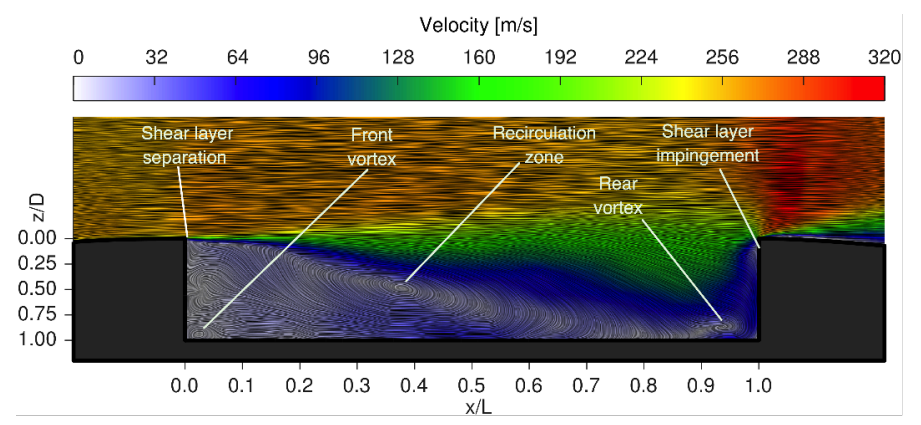

(a) $x-z$ plane, $2 y / W=0.0$

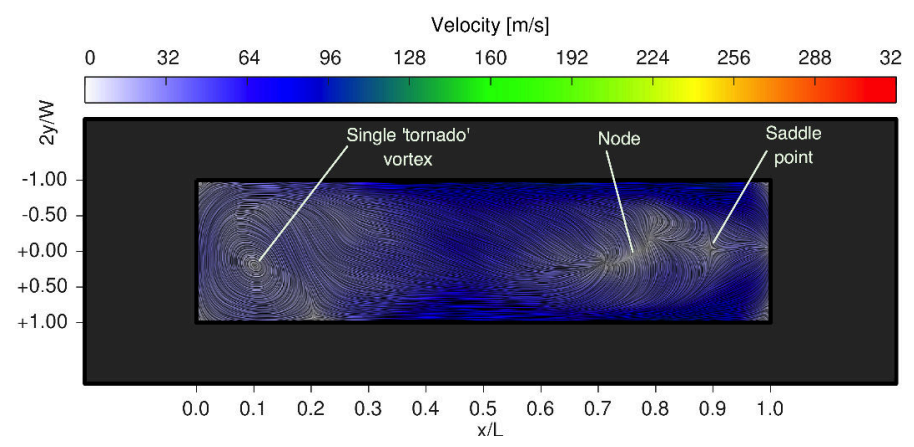

(b) $x-y$ plane, $z / D=0.5$

Figure 10: The mean velocity field at $\alpha=4.5$ degrees; flow from left to right.

front of the cavity but, unlike the reference case, it has a counter-clockwise orientation. Towards the rear of the cavity, at $x / L \approx 0.90$, there is a saddle point. Slightly further upstream there is a node with flow emanating along a short line between $x / L \approx 0.70$ and $x / L \approx 0.80$. The appearance of the saddle and node at this height in the cavity is a consequence of the increased angle of attack whereby flow is moving downstream between the node and saddle but is still moving upstream near the side walls.

At $\alpha=6.0$ degrees (Fig. 11a) the downwards tilt of the main recirculation region's major axis, observed with $\alpha=4.5$ degrees, is maintained and its centroid continues to move forwards to around $x / L=0.33$. The three-eddy structure in the $x-z$ plane, typical of the reference case, remains, however, there is a significant repositioning of the rear-wall stagnation point which is now located at $z / D \approx 0.2$. A strong asymmetry is present at the front of the cavity in the $x-y$ plane (Fig. 11b) where a single counter- 


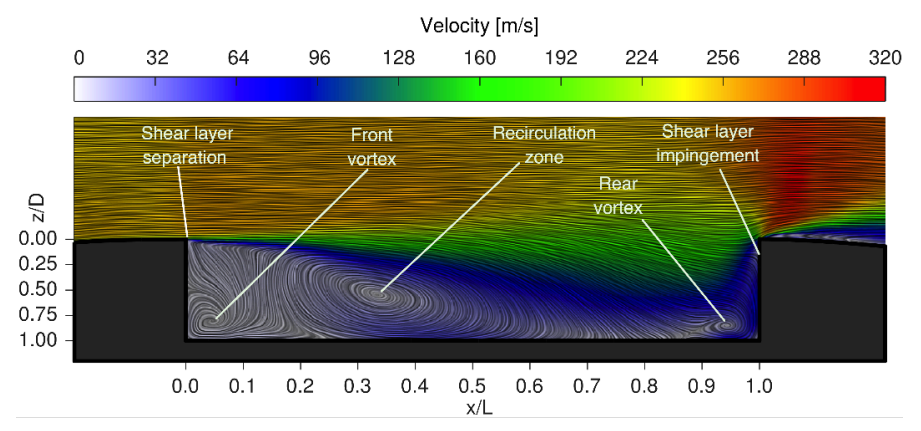

(a) $x-z$ plane, $2 y / W=0.0$

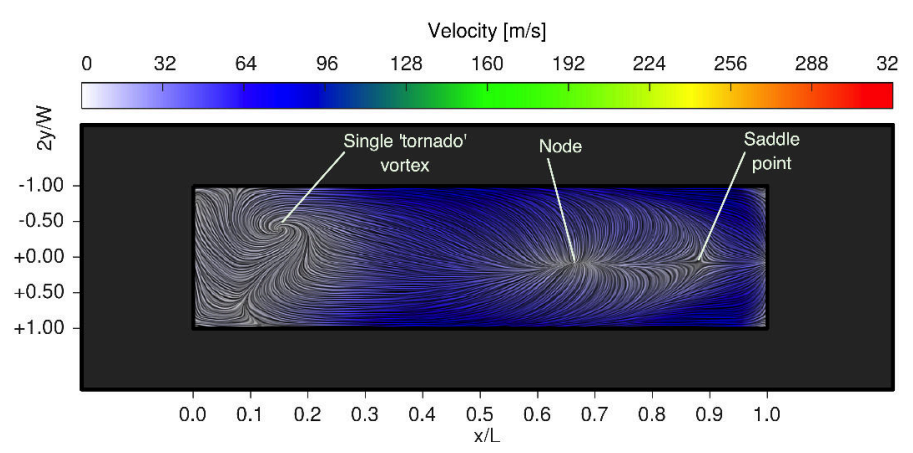

(b) $x-y$ plane, $z / D=0.5$

Figure 11: The mean velocity field at $\alpha=6.0$ degrees; flow from left to right.

clockwise 'tornado' vortex was observed. As with the $\alpha=4.5$ degree case the rear part of the cavity contains a saddle point (at $x / L \approx 0.88$ ) and a node (at $x / L \approx 0.67$ ) from which flow is again emanating. There is a strong upstream flow near the side walls that feeds the tornado vortex. The minimum value of $\overline{C_{p}}$ is again aft of the recirculation zone; in this case by approximately $0.04 x / L$.

Fig. 12 compares the time-averaged streamwise velocity profiles along the cavity centreline, for each of the four angles of attack, at five longitudinal locations $(x / L=0.1$, $0.3,0.5,0.7$ and 0.9$)$. The profiles at $\alpha=0$ degrees are in very good agreement with the results of Lawson and Barakos [12]. Although the freestream velocity was the same in all four cases, the local velocity above the cavity varied due to the change in angle of attack. The front two profiles exhibit very little change with increasing angle of attack, however, the rear three profiles, which are aft of the main recirculation zone, 


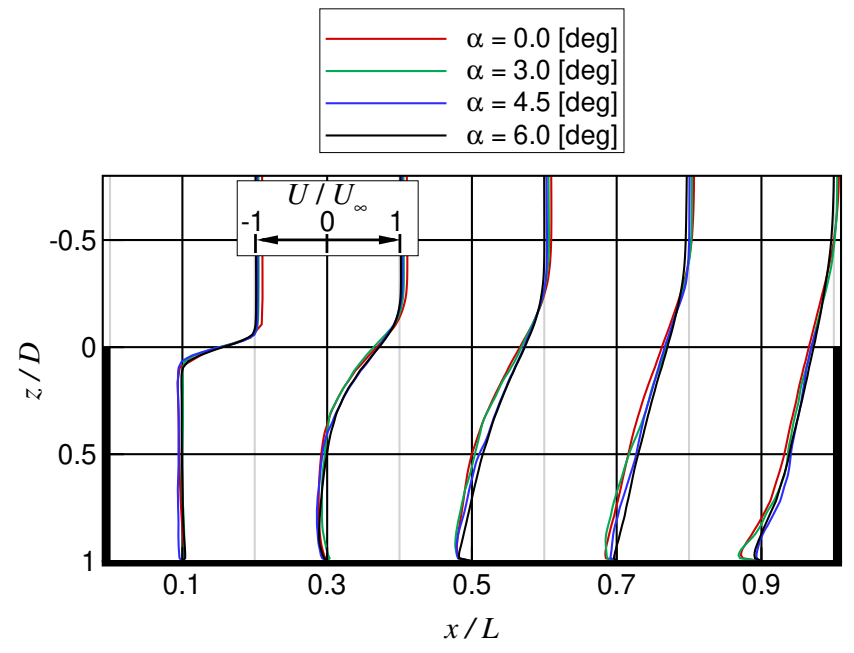

Figure 12: Time-averaged streamwise velocity profiles at four angles of attack $(2 y / W=0)$.

show a generally increasing streamwise velocity within the cavity as angle of attack is increased. This is to be expected as the rear of the cavity is exposed to more of the freestream flow.

\subsection{Non-stationary flow analysis}

Analysis of the OASPL indicated that the rearmost station of the cavity $(x / L=0.9)$ was the position with the highest acoustic loads. It was decided, therefore, to analyse the effect of changes in angle of attack on the SPL calculated in this area. Depending on the angle of attack analysed it was possible to discern up to four Rossiter-Heller modes in the spectra. A summary of the analysis is presented in Table 2.

Fig. 13 shows that, at zero angle of attack, the spectrum was characterised by two resonant modes: M1, the weaker, located at a Strouhal number of 0.2574 with a SPL of $144.6 \mathrm{~dB}$, and M2, the stronger, located at a Strouhal number of 0.6434 with a SPL of $150.5 \mathrm{~dB}$. Both tones were in good agreement with those predicted by Eqn. 1 . Applying Delprat's decomposition rule [8], a fundamental acoustic Strouhal number, $\mathrm{St}_{a}$, of 0.3860 was obtained, with an amplitude modulation parameter, $\xi$, of 0.3331 and a modulating Strouhal number, $\Delta$ St of 0.0134 . The theoretically-predicted values, 
Table 2: Summary of the resonant mode characteristics. Data refers to a pressure probe at $x / L=0.9$, $2 y / W=0.0, z / D=0.0$.

\begin{tabular}{|c|c|c|c|c|c|c|c|c|c|}
\hline$\alpha$ (deg.) & $\mathrm{St}_{a}$ & $\mathrm{St}_{b}$ & $\xi$ & $\Delta \mathrm{St}$ & & $M_{1}$ & $M_{2}$ & $M_{3}$ & $M_{4}$ \\
\hline \multirow{3}{*}{0.0} & \multirow{3}{*}{0.3860} & \multirow{3}{*}{0.1286} & \multirow{3}{*}{0.3331} & \multirow{3}{*}{0.0134} & St & 0.2574 & 0.6434 & - & - \\
\hline & & & & & SPL (dB) & 144.6 & 150.5 & - & - \\
\hline & & & & & PR (\%) & 3.87 & 28.33 & - & - \\
\hline \multirow{3}{*}{3.0} & \multirow{3}{*}{0.3863} & \multirow{3}{*}{0.1504} & \multirow{3}{*}{0.3893} & \multirow{3}{*}{0.0139} & St & 0.2359 & 0.6222 & - & - \\
\hline & & & & & SPL (dB) & 148.2 & 153.0 & - & - \\
\hline & & & & & PR (\%) & 6.22 & 33.03 & - & - \\
\hline \multirow{3}{*}{4.5} & \multirow{3}{*}{0.3615} & \multirow{3}{*}{0.1080} & \multirow{3}{*}{0.2987} & \multirow{3}{*}{0.0268} & St & 0.2535 & 0.6150 & - & - \\
\hline & & & & & SPL (dB) & 144.4 & 146.8 & - & - \\
\hline & & & & & PR (\%) & 6.64 & 25.30 & - & - \\
\hline \multirow{3}{*}{6.0} & \multirow{3}{*}{0.3359} & \multirow{3}{*}{0.0667} & \multirow{3}{*}{0.1987} & \multirow{3}{*}{0.0402} & St & 0.2692 & 0.6051 & 0.9473 & 1.2650 \\
\hline & & & & & SPL (dB) & 146.5 & 147.9 & 139.4 & 137.7 \\
\hline & & & & & PR $(\%)$ & 6.38 & 13.69 & 2.15 & 1.04 \\
\hline
\end{tabular}

using eqns. 3 and 4 , were $\xi=0.3509$ and $\mathrm{St}_{a}=0.3923$. Wavelet analysis was used to determine the persistence, PR, of the modes. The resulting values were $3.87 \%$ for M1, and $28.33 \%$ for M2, confirming the intermittent nature of the resonating modes. This is consistent with previous studies [36].

When the angle of attack was increased to 3.0 degrees two major differences were observed. Firstly, the principal tones, M1 and M2, increased their power levels by $3.6 \mathrm{~dB}$ and $2.5 \mathrm{~dB}$ respectively. Secondly, while the PR index of M2 increased only slightly from $28.33 \%$ to $33.03 \%$, M1 saw a near doubling of this quantity, increasing from the previous $3.87 \%$ to $6.22 \%$. Such a result indicates that the increased angle of attack reinforced the nature of the first resonant mode, making it less intermittent and more stable. Noticeably, the fundamental acoustic Strouhal number and the modulating Strouhal 


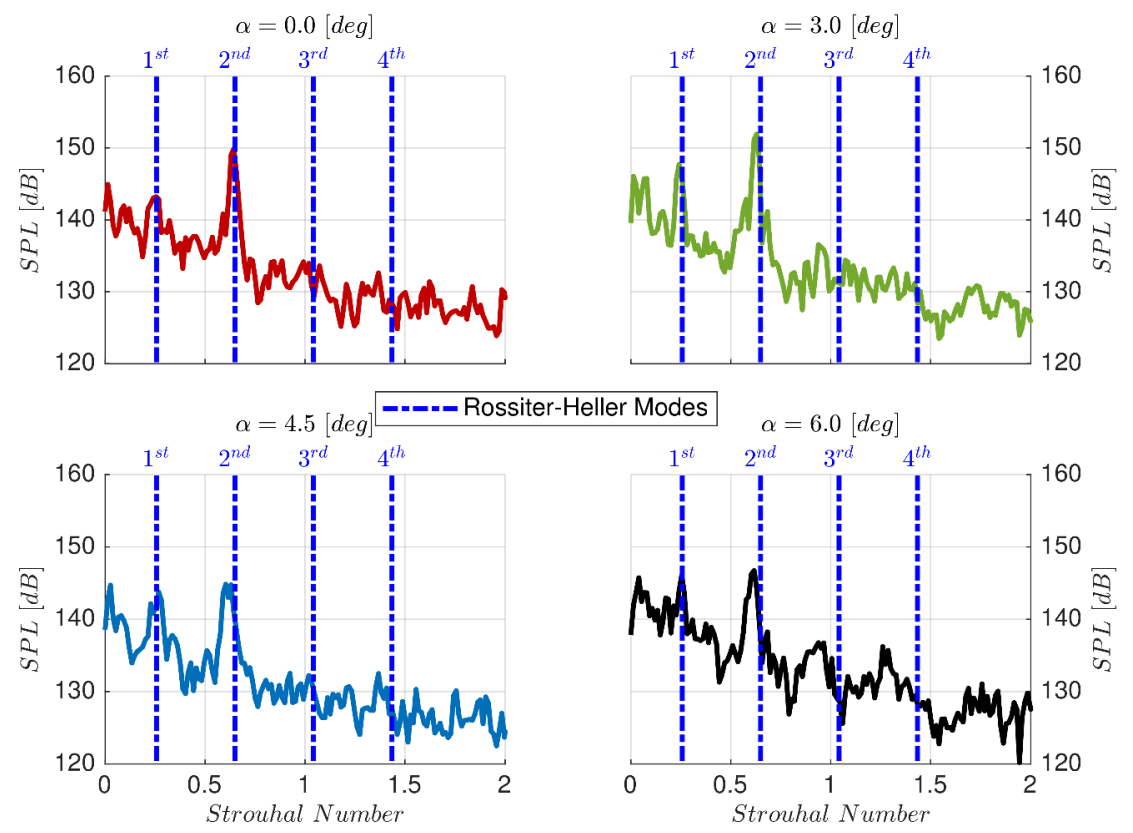

Figure 13: Angle of attack effects on SPL. Data refers to pressure probe located at $x / L=0.9,2 y / W=0.0$, $z / D=1.0$.

number did not show significant change. The amplitude modulation parameter did increase, however, by $17 \%$ to 0.3893 .

At 4.5 degrees angle of attack, two resonant tones, M1 and M2, were once again observed. Table 2 shows that their SPL values decreased with respect to the 3 degree angle of attack case. Delprat's decomposition showed that all parameters underwent a consistent decrease, indicating a re-organisation of the aeroacoustic system, even though the spectrum looked very similar to the 3.0 degrees angle of attack case. At 6.0 degrees angle of attack, the flow was characterised by the appearance of two additional modes, M3 and M4, with very low PR values. Again, Delprat's parameters indicated a change had occurred with respect to the previous angle of attack. These additional tones, due to their low PR values, were barely distinguishable from the background noise. This suggested that such modes might have been present at lower angles of attack; however, their power levels may have been too low to raise them above the background noise spectrum. 
Fig. 14 plots the parameters $\mathrm{St}_{a}, \mathrm{St}_{b}, \xi$ and $\Delta \mathrm{St}$ against the sine of the angle of attack. The carrier frequency, $\mathrm{St}_{a}$, was assumed to have a constant value up to 3.0 degrees angle of attack. Beyond this point, the trend was a linear (with negative slope) function of the sine of the angle of attack. Such a result indicated that a velocity vector component directed towards the $z$-axis of the cavity might be influencing the overall feedback mechanism. As dictated by Eqn. 4, this parameter is only dependent on the freestream conditions, and is correlated to the acoustic feedback loop that the geometry of the cavity creates. As shown previously in the mean flow analysis, an increase in the angle of attack redirected the shear layer towards the interior of the cavity. It also alters the impact point on the rear wall, hence changing the spatial organisation of the feedback loop. Similar to that observed for the carrier frequency, the modulating frequency, $\Delta \mathrm{St}$, did not change when the angle of attack was increased from zero to 3.0 degrees. Beyond this point, the parameter again showed a linear trend (with positive slope) with the sine of the angle of attack. The amplitude modulation frequency, $\mathrm{St}_{b}$, and amplitude modulation parameter, $\xi$, whilst maintaining a linear trend for angles of attack above 3.0 degrees, did not remain constant between the zero to 3.0 degrees angle of attack range. This different behaviour may be correlated to the dependence of $\mathrm{St}_{b}$ and $\xi$ on the depth of the cavity (as shown in Eqn. 3), making them sensitive to small angular variations in the freestream flow.

Further insight into the angle attack effects could be obtained by analysing the spatial trends of each of the modes; this was done through a wavelet transform analysis. At each station in the centreline rake (Rake 1C), the temporal trend of the wavelet spectral power (WSP) was extracted for a particular Strouhal band, centred on the tone of interest (for M1, at an interval from $\mathrm{St}=0.2$ to $\mathrm{St}=0.3$; for $\mathrm{M} 2$, at an interval from $\mathrm{St}=0.6$ to $\mathrm{St}=0.7$; for $\mathrm{M} 3$, at an interval from $\mathrm{St}=0.9$ to $\mathrm{St}=1.0$; and for $\mathrm{M} 4$, at an interval from $S t=1.2$ to $S t=1.3$ ). The results obtained were used to generate the wavelet spatial-temporal analysis for the Strouhal interval selected.

Fig. 15 shows the effect of changes in angle of attack on the M1 characteristics. At an angle of attack of zero degrees it was observed that, in accordance with its low PR index, M1 consisted of time-localised bursts during the temporal evolution of the signal. This characteristic was present at all longitudinal stations, confirming 

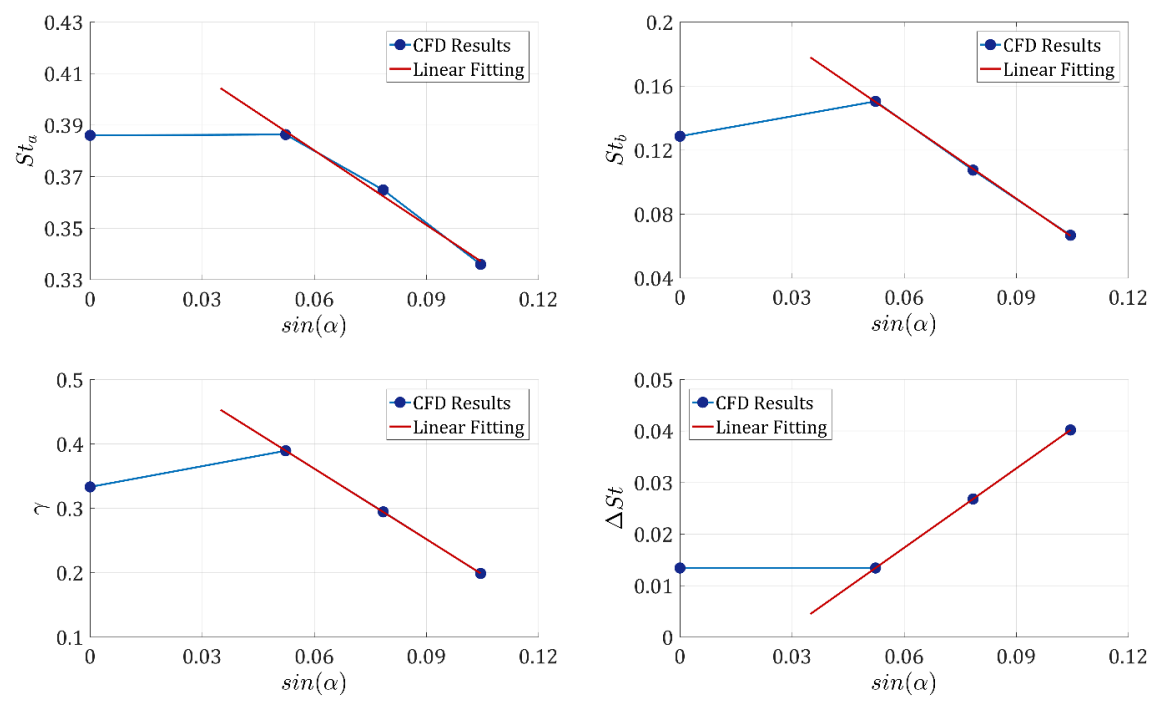

Figure 14: Variation of Delprat's equation parameters with angle of attack. Data refers to a pressure probe located at $x / L=0.9,2 y / W=0.0, z / D=1.0$.

the fluid dynamic shear-layer mode behaviour of the Rossiter-Heller tones. Increasing the angle of attack to 3.0 degrees augmented the acoustic loading experienced by the cavity in this Strouhal band. Nevertheless, the main characteristics were unaffected. The trend was reversed at 4.5 degrees of angle of attack, where the power of the tone decreased, only to increase again when the angle of attack was changed to 6.0 degrees. Nevertheless, no particular effects were observed for the temporal evolution of this tone, and the angle of attack variations did not appreciatively change the time-localised characteristics.

Mode M2, characterised by a regular on/off mechanism (Fig. 16), slightly increased its power and amplitude modulation frequency, when changing from 0.0 to 3.0 degrees angle of attack ${ }^{4}$. Strong differences were observed in the spatial-temporal map at 4.5

\footnotetext{
${ }^{4}$ This outcome could be visually identified from a thickening, along the temporal axis, of the vertical striping in the spatial-temporal maps. Striping indicates alternating high/low power levels along the cavity length. The thickening along the temporal axis corresponded to an increase of the amplitude modulation parameter, i.e. an increase in the amplitude modulation frequency.
} 


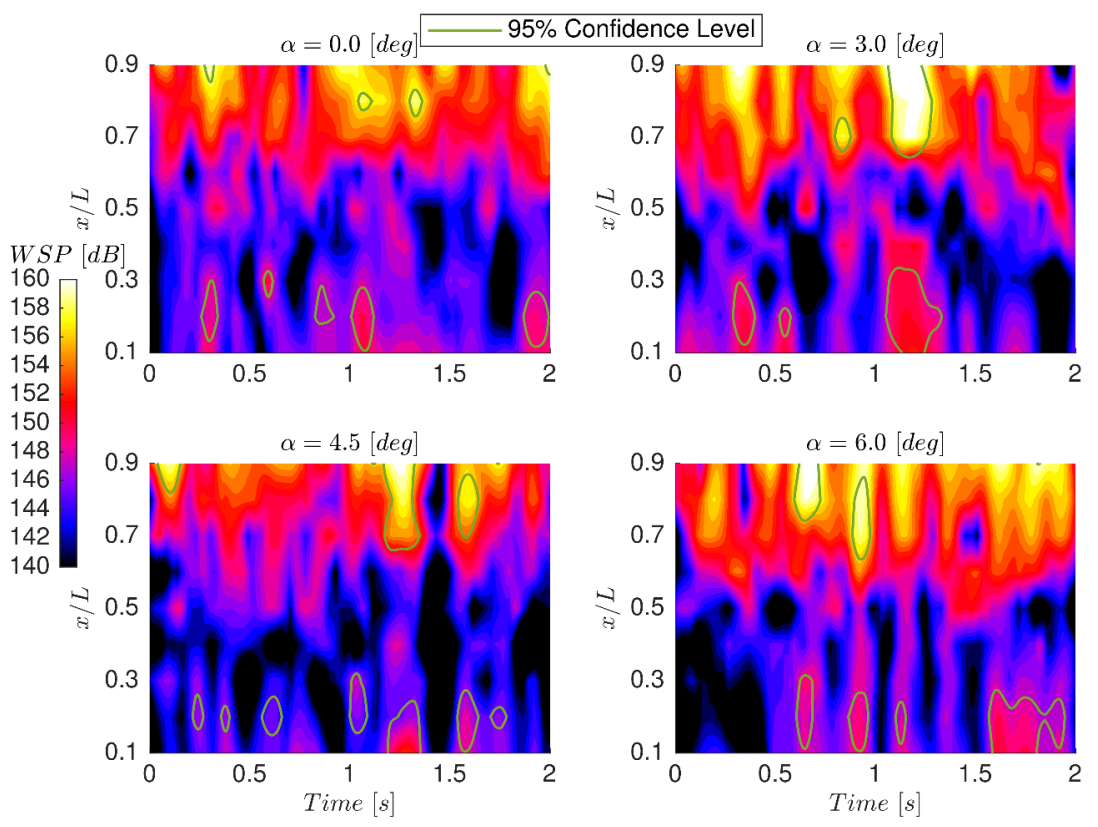

Figure 15: The effect of angle of attack on the WSP for the Strouhal band associated with Rossiter-Heller mode M1 (Rake 1C data with green-coloured contours lines referring to a $95 \%$ Confidence Level threshold).

and 6.0 degrees. In these cases, the tone was characterised by two distinct phases. The first could be called stable, where the temporal trend reflected the lower angle of attack behaviour. The second, which may be referred to as unstable, is characterised by the amplitude modulation of the tone losing its coherence and becoming irregular and intermittent. Noticeably, these phases corresponded to areas in which the wavelet analysis indicated the disappearance of M2. Delprat [8] suggested that temporal irregularities and variations of the amplitude modulator parameter could be responsible for the mode-switching phenomenon, affecting Rossiter-Heller tones. Thus higher angles of attack seem to promote instabilities in the coupling of $\mathrm{St}_{a}$ and $\mathrm{St}_{b}$ leading to the characteristics observed.

The different effects that varying angle of attack had on M1 and M2 might reside in the spatial characteristics of these waves. M1, had a wavelength approximately five times greater than the cavity's length, and was less likely to be influenced by small variations in angle of attack. M2, instead, had a wavelength slightly less than twice 

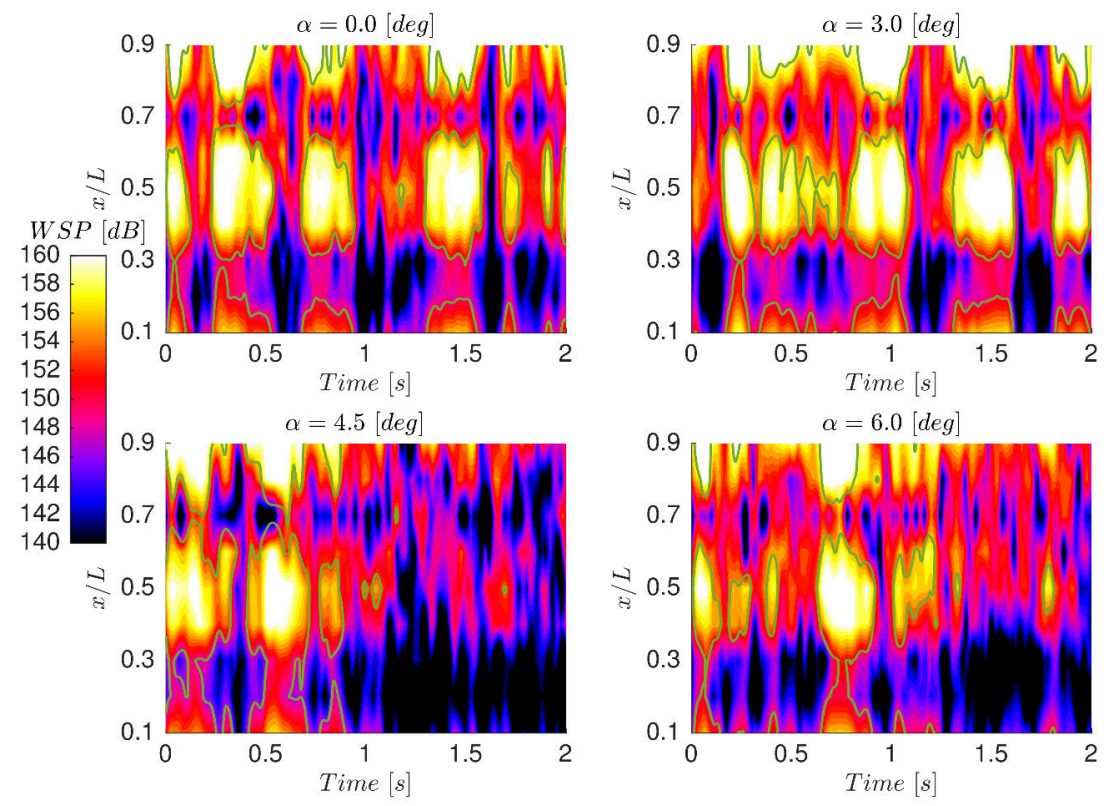

Figure 16: The effect of angle of attack on the WSP for the Strouhal band associated with Rossiter-Heller mode M2 (Rake 1C data with green-coloured contours lines referring to a $95 \%$ Confidence Level threshold).

the cavity length. This characteristically placed the tone antinode coincident with the cavity trailing edge, in a position where it is strongly influenced by any external variation of angle of attack. As shown in the mean flow analysis, at $\alpha=4.5$ degrees, the shear layer trajectory tended to penetrate inside the cavity, thus affecting the M2 characteristics.

Modes M3 and M4 were, according to frequency domain analysis, appeared only at an angle of attack of 6.0 degrees. Nevertheless, the wavelet spatial-temporal maps (Figs. 17 and 18) showed localised bursts of activity, especially at $0.5<x / L<0.7$. Such results indicated that M3 and M4 were always generated, regardless of the angle of attack the cavity was experiencing, but that, only at 6.0 degrees of angle of attack, were such tones capable of gaining enough power to be distinguishable from the background noise in the Fourier spectrum. The low PR indices calculated and shown in Table 2 reinforces the difficulty that these modes had in acquiring coherence during the temporal evolution of the signal. Moreover, the fact that their local maxima were 

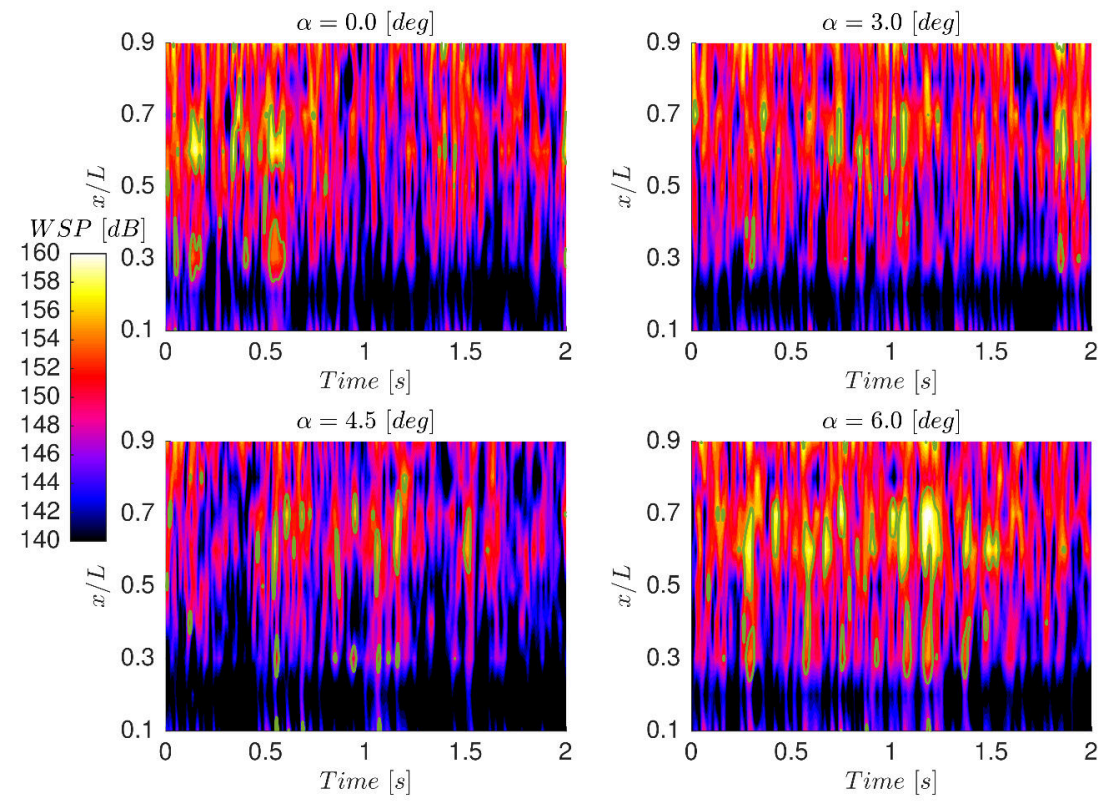

Figure 17: The effect of angle of attack on the WSP for the Strouhal band associated with Rossiter-Heller mode M3 (Rake 1C data with green-coloured contours lines referring to a $95 \%$ Confidence Level threshold).

observed at $x / L \approx 0.7$ (the rearmost boundary of the main recirculation zone) further supported the theory that, only at higher angles of attack, where the flow injection in this area was greater, was enough energy transferred to modes M3 and M4 for them to appear as coherent peaks in the spectrum.

\section{Conclusions}

Cavity flows have been shown to be strongly affected by angle of attack variations, with all mean quantities measured being influenced. The mean pressure coefficient saw an overall rise whenever the angle of attack was increased, however the change was not monotonic. This result correlates generally with the increased vertical component of freestream velocity, thereby increasing the mass flow towards the cavity floor. A particularly unusual effect was observed at an angle of attack of 4.5 degrees, where only the front part of the cavity experienced the mean pressure coefficient rise, with 

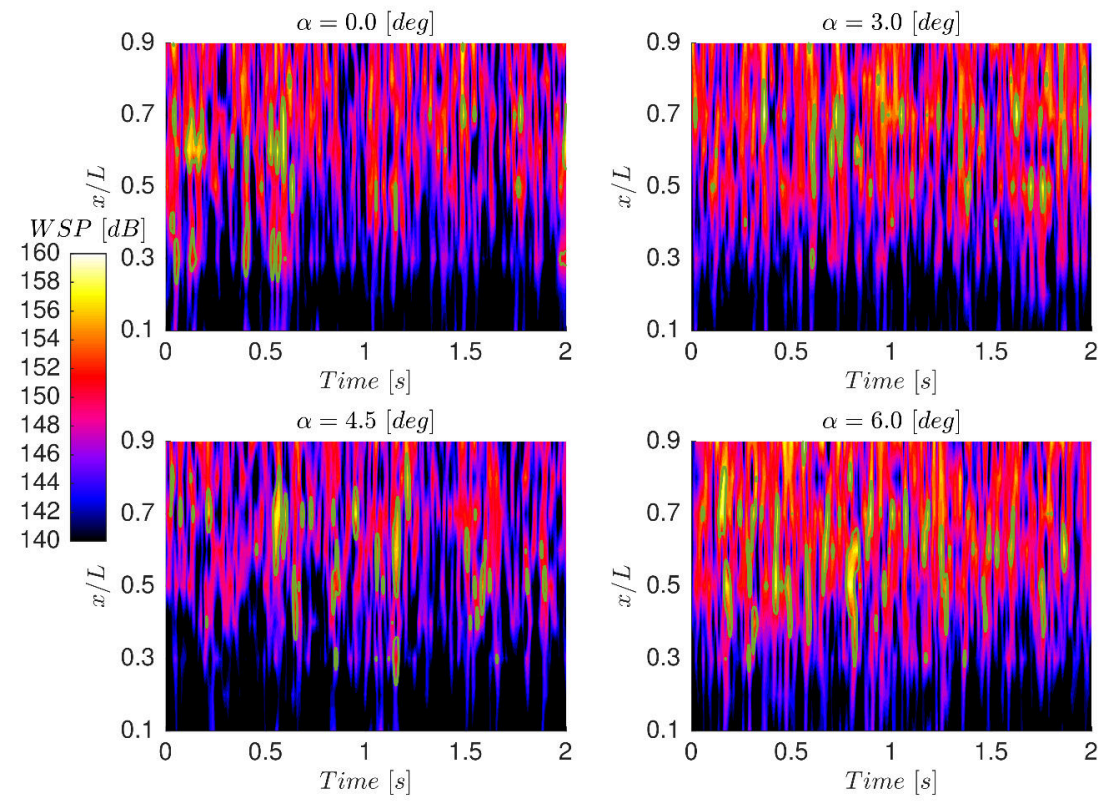

Figure 18: The effect of angle of attack on the WSP for the Strouhal band associated with Rossiter-Heller mode M4 (Rake 1C data with green-coloured contours lines referring to a $95 \%$ Confidence Level threshold).

the rear assuming a value similar to that seen in the 3.0 degrees angle-of-attack case. This observation may be explained with reference to the change in flow configuration and mean velocity vector field experienced inside the cavity at this angle of attack. Of particular note was the reconfiguration of the main recirculation zone, the core moving forwards towards the cavity leading edge. Its shape was also changed, to that of an ellipse, with its major axis rotated by approximately 30 degrees (away from the exit plane at the rear). The measured OASPL values appeared to be highly influenced by this flow phenomenon. The overall noise levels dropped approximately $3 \mathrm{~dB}$ below those of the reference ( $\alpha=0$ degrees) case.

Non-stationary flow analysis also revealed dependence upon angle of attack variations. Spectral analysis indicated that the cavity resonance mechanism was composed of two Rossiter-Heller modes, with Delprat parameters showing a linear trend with the sine of the angle of attack for values between 3.0 and 6.0 degrees. This indicated that the vertical component of the free-stream velocity vector field, oriented in the 
cavity $z$-axis, influenced the fundamental acoustic Shrouhal number, $\mathrm{St}_{a}$, as well as the amplitude modulation parameter, $\xi$, and the frequency modulation parameter, $\Delta$ St. At the highest angle of attack tested, two additional tones appeared, namely the third and the fourth Rossiter-Heller modes. Wavelet analysis indicated that such modes were present at lower angles of attack but at much lower power levels so that they were masked by the background noise. The intermittency of the two main Rossiter-Heller tones could be expressed with the PR index. This indicated that increasing the angle of attack from 0.0 to 3.0 degrees produced an increment in the acoustic load while a further increase tended to affect the resonance mechanism and thereby reduce the coherence and the temporal footprints of the modes.

\section{References}

[1] D. Rockwell and E. Naudasher. Review - self sustaining oscillations of flow past cavities. F. Fluid Eng., 100(2):152-165, 1978.

[2] M. Kompenhans, E. Ferrer, M. Chavez, and E. Valero. Numerical study of three dimensional acoustic resonances in open cavities at high reynolds numbers. Aerosp. Sci. Technol., 45:501 - 511, 2015.

[3] Zhaoxin Ren, Bing Wang, Bowen Hu, and Longxi Zheng. Numerical analysis of supersonic flows over an aft-ramped open-mode cavity. Aerosp. Sci. Technol., 78: $427-437,2018$.

[4] Tanuj Gautam, G. Lovejeet, and Aravind Vaidyanathan. Experimental study of supersonic flow over cavity with aft wall offset and cavity floor injection. Aerosp. Sci. Technol., 70:211 - 232, 2017.

[5] Hongkang Liu, Chao Yan, Yatian Zhao, and Yupei Qin. Analysis of pressure fluctuation in transonic cavity flows using modal decomposition. Aerosp. Sci. Technol., 77:819 - 835, 2018.

[6] J. E. Rossiter. Wind-tunnel experiments on the flow over rectangular cavities at subsonic and transonic speeds. Reports and Memoranda 3438, Aeronautical Research Council, 1966. 
[7] H. Heller, D. Holmes, and E. Covert. Flow-induced pressure oscillations in shallow cavities. F. Sound Vib., 18:545-553, 1971.

[8] N. Delprat. Low-frequency components and modulation processes in compressible cavity flows. F. Sound Vib., 329(22):4797-4809, 2010.

[9] R. L. Clark. Evaluation of F-111 weapon bay aero-acoustic and weapon separation improvement techniques. Technical Report AFFDL-TR-79-3003, Air Force Flight Dynamics Laboratory, Wright-Patterson Air Force Base, OH, February 1979.

[10] C. Kannepalli, C. Chartrand, R. Birkbeck, N. Sinha, and N. Murray. Computational modelling of geometrically complex weapons bays. In 17th AIAA/CEAS Aeroacoustics Conference, number AIAA-2011-2774, Portland, OR, 5-8 June 2011.

[11] M. H. Morton, J. T. Cox, and E. A. Powell. Initial assessment of a CFD aplication for predicting jet fighter aircraft cavity bay acoustics for subsonic and supersonic aircraft states. In 53rd AIAA/ASME/ASCE/AHS/ASC Structures, Structural Dynamics and Materials Conference, number AIAA-2012-1624, Honolulu, HI, 23-26 April 2012.

[12] S. J. Lawson and G. N. Barakos. Evaluation of DES for weapons bays in UCAVs. Aerosp. Sci. Technol., 14(6):397-414, September 2010.

[13] R. A. Chaplin and T. J. Birch. The aero-acoustic environment within the weapons bay of a generic UCAV. In 30th AIAA Applied Aerodynamics Conference, number AIAA-2012-3338, New Orleans, LA, 25-28 June 2012.

[14] B. P. Hallissy and N. S. Hariharan. Prediction of unsteady flow in UCAV weapon's bay using CREATE-AV Kestrel. In 52nd Aerospace Sciences Meeting, number AIAA2014-0751, National Harbor, MD, 13-17 January 2014.

[15] L. S. Ukeiley, M. Sheehan, F. Coiffet, F. Alvi, S. Arunajatesan, and B. Jansen. Control of pressure loads in geometrically complex cavities. F. Aircraft, 45(3):10141024, May-June 2008. 
[16] N. E. Murray and B. J. Jansen. Effect of door configuration on cavity flow modulation process. AIAA f., 50(12):2932-2937, December 2012.

[17] K. M. Casper, J. L. Wagner, S. J. Baresh, J. F. Henfling, R. W. Spillers, and B. O. M. Pruett. Complex geometry effects on cavity resonance. AIAA f., 54(1):320-330, 2016.

[18] P. R. Spalart. Young person's guide to detached-eddy simulation grids. Contractor Report CR-2001-211032, NASA, Langley Research Center, Hampton, VA, July 2001.

[19] V. Thangamani, K. Knowles, and A. J. Saddington. The effects of scaling on high subsonic cavity flow oscillations and control. F. Aircraft, 51(2):424-433, 2014.

[20] F. R. Menter. Best Practice: Scale-Resolving Simulations in ANSYS CFD. ANSYS Inc., 2 edition, November 2015.

[21] D. C. Wilcox. Turbulence Modeling for CFD. DCW Industries, 3rd edition, 2006. ISBN: 978-1928729082.

[22] ANSYS. ANSYS Fluent 16 theory guide, 2016.

[23] F. R. Menter. Two-equation eddy-viscosity turbulence models for engineering applications. AIAA f., 32(8):1598-1605, 1994.

[24] M. L. Shur, P. R. Spalart, M. Kh. Strelets, and A. K. Travin. A hybrid RANS-LES approach with delayed-DES and wall-modelled LES capabilities. Int. f. Heat Fluid Fl., 29(6):1638-1649, December 2008.

[25] M. B. Tracy and E. B. Plentovich. Characterization of cavity flow fields using pressure data obtained in the Langley 0.3-meter transonic cryogenic tunnel. Tech. Rep. TM-4436, NASA Langley Research Center, Hampton, VA, USA, March 1993.

[26] A. K. M. Fazle Hussain. Coherent structures and turbulence. F. Fluid Mech., 173: 303-356, December 1986.

[27] D. Bacci, A. J. Saddington, and D. Bray. Identification of the formation of resonant tones in compressible cavity flows. Aerosp. Sci. Technol., 77:320-331, 2018. 
[28] P. D. Welch. The use of fast Fourier transform for the estimation of power spectra: A method based on time averaging over short, modified periodograms. IEEE T. Acoust. Speech, 15(2):70-73, June 1967.

[29] H. H. Bruun. Hot-Wire Anemometry Principles and Signal Analysis. Oxford University Press, Oxford, UK, 1996.

[30] D. E. Newland. An Introduction to Random Vibrations, Spectral \& Wavelet Analysis. Longman, Harlow, UK, 1993.

[31] C. Torrence and G. P. Compo. A practical guide to wavelet analysis. Amer. Meteor. Soc., 79(1):61-78, 1998.

[32] R. D. Knowles, M. V. Finnis, A. J. Saddington, and K. Knowles. Planar visualization of vortical flows. P. I. Mech. Eng. G-7. Aer., 220(G6):619-627, 2006. ISSN 0954-4100.

[33] S. D. Crook, T. C. W. Lau, and R. M. Kelso. Three-dimensional flow within shallow, narrow cavities. F. Fluid Mech., 735:587-612, 2013.

[34] B. Khanal, K. Knowles, and A. J. Saddington. Computational study of flowfield characteristics due to presence of stores in cavities. Aeronaut. f., 115(1172):669681, November 2011. doi: 10.1017/S0001924000006394.

[35] B. Khanal, K. Knowles, and A. J. Saddington. Computational investigation of cavity flow control using a passive device. Aeronaut. f., 116(1176):153-174, February 2012. doi: $10.1017 / S 0001924000006679$.

[36] M. A. Kegerise, E. F. Spina, S. Garg, and L. N. Cattafesta. Mode-switching and nonlinear effects in compressible flow over a cavity. Phys. Fluids, 16(3):678-687, 2004. 